\title{
GM1 asymmetry in the membrane stabilizes pores
}

\author{
Mina Aleksanyan ${ }^{1}$, Rafael B. Lira ${ }^{1,2}$, Jan Steinkühler ${ }^{1,3}$ and Rumiana Dimova ${ }^{1 *}$ \\ ${ }^{1}$ Department of Colloid Chemistry, Max Planck Institute of Colloids and Interfaces, 14424 Potsdam, Germany. \\ ${ }^{2}$ Present address: Moleculaire Biofysica, Zernike Instituut, Rijksuniversiteit, Groningen, the Netherlands \\ ${ }^{3}$ Present address: Department of Biomedical Engineering, Northwestern University, Evanston, IL 60657, USA
}

\begin{abstract}
Cell membranes are highly asymmetric and their stability against poration is crucial for survival. We investigated the influence of membrane asymmetry on electroporation of giant vesicles with membranes doped with GM1, a ganglioside asymmetrically enriched in neuronal cell membranes. Compared to symmetric membranes, the lifetimes of micronsized pores are about an order of magnitude longer suggesting that pores are stabilized by GM1. Internal membrane nanotubes caused by the GM1 asymmetry, obstruct and additionally slow down pore closure, effectively reducing pore edge tension and leading to leaky membranes. Our results point to the drastic effects this ganglioside can have on pore resealing in biotechnology applications based on poration as well as on membrane repair processes.
\end{abstract}

Key words: giant vesicles, membrane asymmetry, electroporation, membrane stability, membrane permeation, GM1, membrane nanotubes, macropores

\section{Introduction}

Pores in membranes allow for exchange and introduction of substances in cells, and when generated exogenously, their closure is crucial for cell survival. Among the different approaches of generating pores ${ }^{1}$, electroporation offers the attractive feature of precise localization and temporal control. Thus, electroporation-based techniques have gained significant importance in biotechnology over the years as one of the low-cost, safe, practical and efficient ways of accessing the inner compartments of cells and controlling biological processes..$^{2-5}$ Depending on the duration, strength and number of electric pulses, membrane electroporation can either result in a permanent cell lysis (i.e. irreversible electroporation), or temporary poration followed by membrane resealing (i.e. reversible electroporation) ${ }^{4}$ Irreversible electroporation has broad applications in regenerative medicine ${ }^{6}$ and tissue engineering ${ }^{7}$ including tumor ablation ${ }^{8}$, electrochemotherapy ${ }^{9,10}$ and exogenous cell engraftment ${ }^{11}$. On the other hand, reversible electroporation has been one of the most widely applied methods in biomedical engineering ${ }^{12}$ including anticancer treatment ${ }^{13}$, gene and drug delivery $^{14}$, cell transfection ${ }^{15}$ and inactivation of microorganisms ${ }^{16}$. Electroporation thresholds and pore kinetics are known to differ from cell to cell and depend on a large variety of parameters including pulse shape, duration, number and repetition, cell size and state as well as environmental conditions. ${ }^{17}$ Despite the numerous theoretical and experimental studies on electroporation (see e.g. refs. ${ }^{18-20}$ ), the fundamental mechanisms underlying the plasma membrane resealing after an electrical breakdown have not been yet fully explained; for example, it remains unclear why pores have a wide range of lifetimes spanning from milliseconds to minutes ${ }^{21-23}$. However, understanding the detailed membrane reorganization and pore stability is crucial for the optimization of clinical settings of electroporation as well as membrane repair and wound healing.

To resolve the underlying mechanisms of poration and stability of plasma membranes, giant unilamellar vesicles (GUVs) ${ }^{24}$,
${ }^{25}$ as cell-size simple membrane systems are commonly investigated $^{26-28}$ because the membrane response is directly accessible via optical microscopy. Electroporation is initiated by the increase in membrane tension induced by the electric field. Above the membrane electroporation threshold, pores are formed, relaxing the tension. ${ }^{29,}{ }^{30}$ The pores can spontaneously reseal driven by membrane edge tension, ${ }^{31-33}$ the energetic cost of lipid rearrangement along the pore rim. Not surprisingly, the edge tension depends on membrane composition as well as on the medium (presence of ions, molecules or detergents). ${ }^{34-36}$

The response of single-component (symmetric) GUVs to electric fields has been thoroughly explored. ${ }^{26,} 27,30$ For such simple membranes, the application of a single DC pulse can lead to GUV deformation and formation of micron-sized pores (macropores). The lifetime of these pores is on the order of a few hundreds of milliseconds. ${ }^{30,37}$ However, very simple model membranes might not sufficiently well represent the response of the complex plasma membrane, which exhibits both sophisticated composition and leaflet asymmetry. Here, we explore the effect of asymmetry albeit in a simple model membrane, namely one made of palmitoyloleoylphosphocholine (POPC) and doped with the ganglioside GM1. GM1 is involved in many biological events as one of the major components of the outer leaflet of the mammalian membranes. ${ }^{38,}{ }^{39}$ In addition, it is asymmetrically distributed and abundant in the nervous system and is associated with neuronal differentiation and development processes. ${ }^{40} \mathrm{GM} 1$ asymmetry in GUV membranes was found to induce substantial membrane curvature, leading to the formation of membrane nanotubes ${ }^{41,42}$ In addition, the lifetime of electro-induced pores in GM1-doped GUVs was found to be orders of magnitude longer than pores formed on typical (and symmetric) POPC GUVs. ${ }^{30}$ This naturally raises the question of whether it is just the presence of GM1 or also its asymmetric distribution in the membrane that dramatically slows down pore closure. Answering this question can shed light on the stability of cells when porated (not only via electric fields) and help resolve mechanisms of plasma membrane repair. Thus, we aimed 

available under aCC-BY 4.0 International license.

at investigating in detail the resealing dynamics of electroporated GM1-doped GUVs both as a function of GM1 fraction and membrane asymmetry.

\section{Experimental Section}

Vesicle preparation. GUVs were electroformed from $4 \mathrm{mM}$ of POPC and $0-4 \mathrm{~mol} \% \mathrm{GM}$ in $1 \mathrm{mM}$ HEPES. For fluorescence imaging of vesicles, $0.1 \mathrm{~mol} \%$ 1,2-dipalmitoyl-sn-glycero-3-phosphoethanolamine-N-(lissamine rhodamine B sulfonyl) (DPPE-Rh) was added. For the assessment of GM1 incorporation into the membrane, Alexa-labelled cholera toxin $\mathrm{B}$ (CTB-Alexa) was added to the vesicle solution, see section S1 in the supporting information (SI) for details. For the generation of GM1 leaflet asymmetry, GM1-doped GUVs were 10-fold diluted in isotonic $1 \mathrm{mM}$ HEPES buffer, see SI section S2. For long-term permeation studies, the GUVs were electroformed in $200 \mathrm{mM}(200 \mathrm{mOsmol} / \mathrm{kg})$, diluted in isotonic glucose solution and, subsequently, calcein was added to a final concentration of $5 \mu \mathrm{M}$.

Experimental methods. POPC vesicles doped with $0-4$ mol\% GM1 with symmetric or asymmetric membrane were subjected to a single direct current (DC) pulse $(0.3-0.6 \mathrm{kV} / \mathrm{cm}$, 3 or $50 \mathrm{~ms}$ ) and their responses were recorded either with confocal microscopy, or under epifluorescence or phase contrast combined with high speed imaging, see SI section S3. The membrane edge tension was deduced from analysis of the pore closure, ${ }^{43}$ see SI section S6. For the analysis of long-term permeation, GUVs were exposed to a single DC pulse and calcein entry was monitored for 5 minutes. Quantification of GUV leakage was performed through fluorescence intensity analysis, see SI section S7. In all experiments, microscopy images were analyzed either with LASX software or ImageJ. All the datasets were analyzed and plotted with Origin Pro software.

\section{Results and Discussion}

Dilution of GM1-doped vesicles results in membrane asymmetry. POPC GUVs doped with 0,2 or 4 mol\% GM1 were prepared in $1 \mathrm{mM}$ HEPES buffer and successful incorporation of GM1 into the membrane was confirmed by binding of CTB-Alexa; see SI section S1 and Fig. S1. The explored GM1 concentrations fall in the range found in neurons. ${ }^{44}$ As previously reported, GM1 in the bilayer is in dynamic equilibrium with GM1 in the surrounding solution. ${ }^{41}$ Thus, the GM1 concentration in the membrane can be controlled by the concentration of free GM1 in the bulk. Because flip-flop of GM1 molecules is negligible on the experimental time scales, the GM1 concentration outside the vesicle can be used to control GM1 leaflet asymmetry. In our experiments, the GUVs were 10-fold diluted in GM1-free isoosmolar buffer, which resulted in the desorption of a large fraction of the GM1 lipids from the outer membrane leaflet, increasing the asymmetry compared to the inner leaflet (Fig. 1A). For example, for vesicles prepared with $2 \mathrm{~mol} \% \mathrm{GM} 1$, the dilution step results in ganglioside concentration of $0.47 \mathrm{~mol} \%$ in the outer leaflet and $1.98 \mathrm{~mol} \%$ in the inner leaflet as characterized previously. ${ }^{41}$ A direct consequence of this asymmetry is the generation of spontaneous (preferred) membrane curvature. ${ }^{45,} 46$ In vesicles with excess area (small volume-to-area ratio), this spontaneous curvature stabilizes vesicle morphologies with highly curved membrane nanotubes, as demonstrated previously. ${ }^{41,47,48}$ In the case of the 10fold diluted vesicles initially prepared with 2 or 4 mol\% GM1, the asymmetric distribution of the ganglioside results in negative spontaneous curvature between around $-1 /(500 \mathrm{~nm})$ and $-1 /(200 \mathrm{~nm})^{41}$ which stabilizes inward nanotubes, see SI section S2 and Fig. S2. In contrast, symmetric vesicles (no dilution) do not form tubes (Fig. S2). In order to assess the membrane stability and the edge tension of symmetric and asymmetric membranes, we applied electric pulses to GUVs of asymmetric or symmetric membranes at varying GM1 concentrations.

Fig. 1. Poration of GUVs with symmetric and asymmetric membranes. (A) Upon dilution, GM1 from the outer GUV leaflet de-

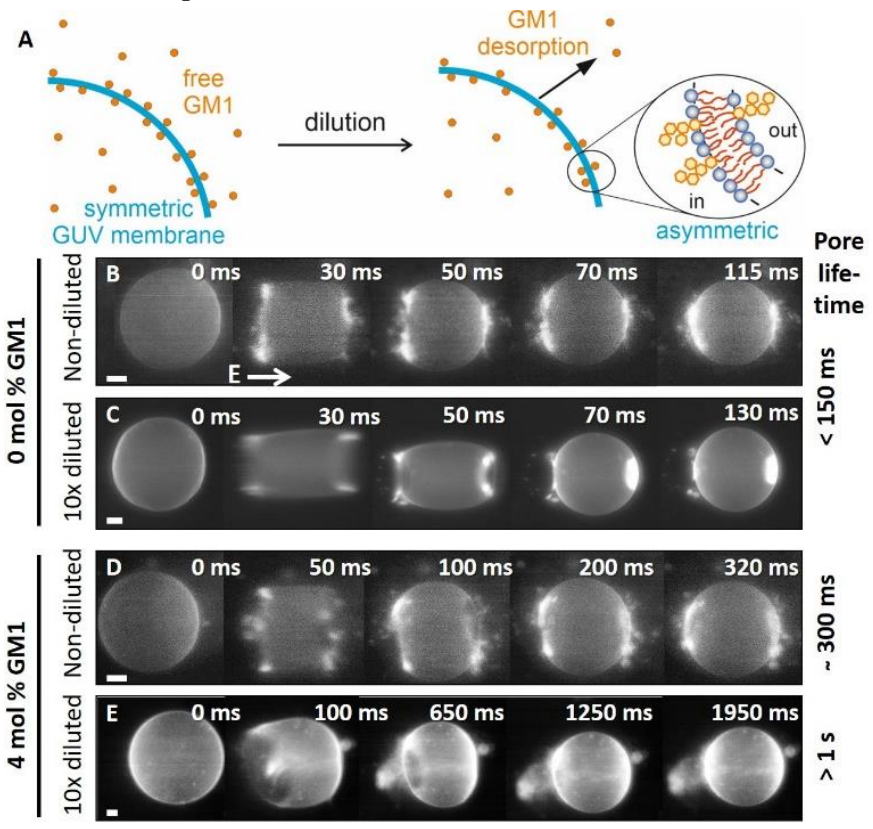

sorbs and renders the membrane asymmetric as illustrated in the sketch. (B-E) Comparison of electroporation of POPC GUVs containing no GM1 (B, C) and 4 mol\% GM1 (D, E) in non-diluted external solution $(\mathrm{B}, \mathrm{D})$ and upon 10 -fold dilution in isotonic external solution $(\mathrm{C}, \mathrm{E})$ imaged with epifluorescence microscopy. The membrane was stained with $0.1 \mathrm{~mol} \%$ DPPE-Rh. The vesicles were exposed to a single DC pulse with amplitude of $0.3-0.4 \mathrm{kV} / \mathrm{cm}$ and duration of $50 \mathrm{~ms}$. The direction of the electric field is illustrated with the arrow in (B). The timestamps in the top-right corner of each snapshot show the time after the beginning of the pulse. The approximate lifetime of the detected micronsized pores are indicated on the right. The sequence in (E) corresponds to Movie S1 in the SI. Scale bars: $10 \mu \mathrm{m}$.

Both GM1 fraction and asymmetry affect pore stability. Pure POPC and 4 mol\% GM1-doped GUVs in both non-diluted and diluted solution were exposed to a single, strong electric pulse (50 ms duration, amplitude of 0.3-0.4 kV/cm), Fig. 1; see also SI section S3. Such pulses raise the transmembrane potential above the poration threshold and optically detectable pores (macropores) are created ${ }^{30}$. The pore dynamics was monitored via high-speed imaging (SI section S3). POPC GUVs exhibit symmetric membranes in both diluted and non-diluted solutions and the pores developed in these membranes had short lifetimes around $150 \mathrm{~ms}$ (Fig. 1B,C) consistent with previous reports. ${ }^{30}$ ${ }^{37}$ On the contrary, non-diluted GUVs symmetrically doped with 4 mol\% GM1 exhibited pores with twice longer lifetime on the order of $300 \mathrm{~ms}$, Fig. 1D.

The above results (Fig. 1) demonstrate that pores are stabilized by GM1 in the membrane. We then investigated the effect of leaflet asymmetry by comparing diluted (asymmetric) and non-diluted (symmetric) GM1-doped vesicles (see sketch in Fig. 1A). Remarkably, in the asymmetric GUVs, as shown in Fig. 1E, pore lifetimes increase dramatically to 1.5 s, i.e. 5 times longer than that of symmetric GM1-doped GUVs and 10 times longer than that for pure-POPC membranes. These findings 
bioRxiv preprint doi: https://doi.org/10.1101/2022.01.21.477228; this version posted January 23, 2022. The copyright holder for this preprint (which was not certified by peer review) is the author/funder, who has granted bioRxiv a license to display the preprint in perpetuity. It is made available under aCC-BY 4.0 International license.

show that not only the presence of GM1 but also leaflet asymmetry stabilize very long-living membrane pores. It is important to note that for the asymmetric membranes, poration was associated with expelling inward tubes through the macropores, Fig. $1 \mathrm{E}$ and SI Movie S1, Fig. S4 and Movie S3. We now discuss three factors for pore stabilization in asymmetric membranes.

(i) Prior to the pulse, inward membrane nanotubes are present in a large fraction of the vesicles (Fig. S2A, B); note that the preparation protocol results in vesicles of different volume-toarea ratio and thus the excess area for tube formation in each vesicle is different. During poration, water flow caused by the higher internal (Laplace) pressure drags nanotubes out through the formed pores (Fig. 2A, SI Movie S2). The tubes protrude from the vesicle interior (Fig. 1E, SI Movie S1) and occasionally also around the pore rim (Fig. 2A, SI Movie S2). Thus, the steric hindrance of the nanotubes on the closing membrane plausibly contributes to increased pore lifetime, also leading to incomplete membrane resealing as shown below.

(ii) Pore lifetime is strongly modulated by the spontaneous curvature of asymmetric membranes. A key observation is that during pore opening, in contrast to symmetric POPC GUVs, asymmetric GM1 vesicles exhibit sprouting of new membrane nanotubes, not present prior to the pulse, (SI Figs. S4, S5, Movies S3, S4). This occurs almost immediately after poration, indicating that the outer spherical membrane segment of the vesicle prefers to rearrange into highly bent nanotubes. This process can be understood considering the membrane spontaneous curvature. Symmetric membranes have zero spontaneous curvature, $m=0$. For the asymmetric membranes $m$ is around $1 / 460 \mathrm{~nm}^{-1}$ for vesicles prepared with $2 \mathrm{~mol} \%$ GM1 and $-1 / 220$ $\mathrm{nm}^{-1}$ for 4 mol\% GM1. ${ }^{41}$ Asymmetric vesicles minimize their bending energy by forming cylindrical nanotubes with radius of $1 /(2 m) .{ }^{49}$ Before poration (in intact vesicles), the ratio of membrane area stored in tubes to area of the weakly curved outer GUV membrane is set by the osmotically stabilized vesicle volume and total membrane area. However, when the volume constraint is relaxed by membrane poration, resealing of the vesicle pore competes for membrane area with formation of new nanotubes. The latter process reduces the vesicle surface area acting analogously to surface tension ${ }^{45}$ that pulls the pore open. Considerations of pore and membrane elastic energy suggest that the asymmetry should result in transforming all available area into nanotubes when a pore of radius $r>r_{c} \equiv$ $\gamma / 2 \mathrm{~km}^{2}$ is formed (SI section S5), where . Introducing the experimentally measured values for the edge tension $\gamma$, the bending rigidity $\kappa$ and $m$ we obtain that pores larger than $15 \mu \mathrm{m}$ ( $2 \%$ mol GM1) or $1.4 \mu \mathrm{m}$ (4\%mol GM1) should become unstable and expand while transforming the membrane into tubes. Typical pore sizes, particularly for the $4 \% \mathrm{~mol} \mathrm{GM} 1$ vesicles were larger and indeed membrane tubulation upon poration was frequently observed for asymmetric GUVs. Almost complete transformation of the vesicle membrane area to a tubular network was also observed occasionally (Fig. S6, Movie S5). However, for most of the vesicles, the period of delayed pore closure and nanotube formation lasts only a few hundred microseconds. Eventually, the membrane pore starts to reseal, which indicates that during pore opening some of the membrane asymmetry is lost, and this corresponds to reduction of the spontaneous curvature $m$. Because of the quadratic relation between critical pore radius $r_{c}$ and spontaneous curvature $m$, a rather small exchange or loss of GM1 is sufficient to enhance pore closure. One possible mechanism for loss of GM1 asymmetry is the interleaflet exchange of membrane-bound GM1 across the pore edge. Another mechanism leading to suppression of asymmetry (i.e. decrease of $m$, ultimately leading to pore closure) is the desorption of GM1 from the inner vesicle leaflet as the enclosed GUV solution now becomes diluted when the pore opens.

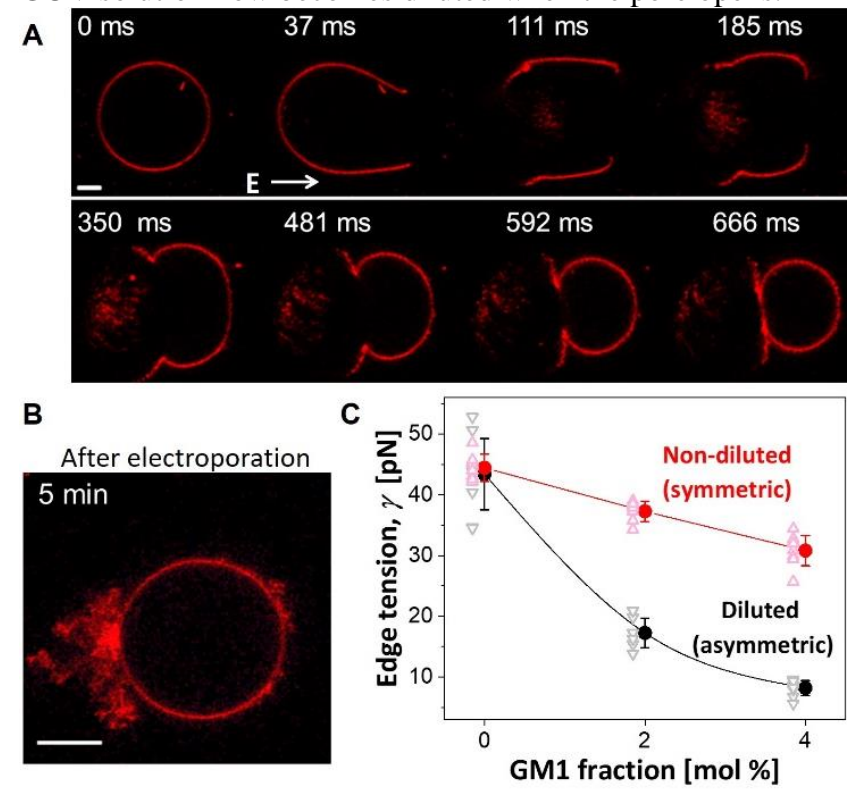

Fig. 2. Electroporation and edge tension of symmetric and asymmetric membranes. (A) A typical example of electroporation of a 4 mol\% GM1-doped asymmetric GUV observed with confocal microscopy upon application of a DC pulse $(0.3 \mathrm{kV} / \mathrm{cm}, 50 \mathrm{~ms})$. The time is relative to the beginning of the pulse. The membrane contains $0.1 \mathrm{~mol} \%$ DPPE-Rh and the GUV was 10 -fold diluted with $1 \mathrm{mM}$ isotonic HEPES buffer. Image sequence corresponds to Movie S2. (B) Cross section of the vesicle 5 minutes after the pulse application. Scale bars: $20 \mu \mathrm{m}$. (C) Edge tension versus GM1 fraction for symmetric and asymmetric GUVs. 6 to 10 vesicles per composition were measured as shown in gray and pink triangles. Mean values and standard deviation of the edge tension are indicated with solid circles and bars (see also Fig. S7). The curves are a guide to the eyes.

(iii) Additionally, the finite membrane viscosity or steric constraints might limit excessive nanotube formation and complete vesicle destabilization.

In summary, we correlate the long pore lifetimes to steric hindrance by nanotubes protruding through the pore and altered membrane mechanics due to dynamic changes in membrane asymmetry during pore closure.

Pore edge tension is lowered by GM1. Next, we set to quantitatively explore the dynamics of pore closure in asymmetric and symmetric vesicles and deduce their pore edge tension using a previously reported method ${ }^{43}$ (for details see SI section S6 and Fig. S7). The measured edge tension of pure POPC GUVs (Fig. 2C) fall in the range of literature values ${ }^{35,36}$ corroborating the consistency of our data and analysis. With increasing GM1 fraction, the mean values of edge tensions obtained from different preparations decrease, see Fig. 2C and SI Table 1. For the asymmetric GM1-doped GUVs, the measured edge tension is an apparent one because of the presence of tubes in the pore area which is not accounted for by the theoretical model ${ }^{31}$. The results for symmetric GUVs scale linearly with GM1 fraction; more GM1 causes stabilization of pores and lower values of edge energy.

This effect in symmetric membranes might be understood considering the cone-like shape of GM1, which could favorably 
locate at the pore rim where the monolayer curvature is high. This molecular effect should be distinguished from the apparent reduction of edge tension by membrane asymmetry detailed above, even though both effects contribute to increasing pore lifetime.

Finally, we return to the membrane remodeling events that were observed during poration of asymmetric GM1 vesicles with nanotubes (Fig. 2A-B, Movies S1, S2). During pore expansion, the electric field and the flow of the solution leaking out orient and extend the tubes out towards the vesicle exterior. As the pore then proceeds to close, it bundles together the protruding tubes resulting in high fluorescence from accumulated membrane material in the form of tubes and small buds at the location of the closed pore. We investigated the influence of this accumulation on the long-term permeability of the vesicles. Here, we distinguish the optically resolvable "macropores" from small "submicroscopic" pores not detected optically.

Asymmetric vesicles become leaky after macropore closure. To test long-term membrane permeation, the vesicles were grown in sucrose solution and diluted in isotonic glucose solution, see SI section S7. As a result of the different refractive index of the sugar solutions, the vesicles appear dark on a brighter background when observed under phase contrast microscopy (SI Fig. S8A). During prolonged observations, we noticed that GM1-doped GUVs lost their optical contrast after macropore closure (Fig. S8), indicating exchange of solution between GUV interior and exterior even after macropore closure. We thus explored whether and to what extent GM1 present in the membrane makes the vesicles leaky. To quantitatively monitor membrane permeability, calcein, a small water-soluble and membrane-impermeable dye, was introduced in the external media (at $5 \mu \mathrm{M}$ ) prior to the application of the pulse. When the membrane is intact, calcein is excluded from the interior of the GUVs, which appear dark in confocal cross sections. The fluorescence dye signal from the vesicle interior upon the application of a single strong DC pulse was used as a measure of prolonged membrane permeability. Calcein permeation in the vesicles was quantified by normalizing the internal fluorescence intensity inside a single GUV 5 minutes after macropore closure by the initial fluorescence intensity right after the macropore closure and the average external fluorescence intensity of the medium (SI section S7), thus eliminating contributions from differences in GUV size, background fluorescence and bleaching. No calcein was detected to flow inside the GUVs while macropores were open leaving the GUV interior black (Fig. 3A). GM1-free (pure POPC) GUVs remained impermeable to calcein $5 \mathrm{~min}$ after electroporation, indicating that pores in these membranes close completely and the membrane reseals. In contrast, asymmetric GM1-containing GUVs became permeable as observed by calcein leaking inside and the fraction of permeable GUVs increasing with GM1 fraction (Fig. 3). These results demonstrate that long-living submicroscopic pores are present in the GM1- doped membranes.

Permeability has been observed to dramatically increase when approaching the main phase transition temperature of the membrane $^{50,51}$. Vesicles containing GM1 have been shown to exhibit gel-like domains ${ }^{52}$ but at fractions higher than those examined here (above $\sim 5 \mathrm{~mol} \%$ ), which is why we can exclude this mechanism of increased permeability. The GM1-doped vesicles are also not leaky in the absence of electroporation (they preserve their sugar asymmetry over a period of at least 3 hours).

A plausible mechanism for stabilizing the submicroscopic pores causing long-term leakage could be steric obstruction by accumulated GM1 at the pore rims as well as protruding nanotubes. Indeed, GM1 bearing a single negative charge could be accumulated at the vesicle poles during the application of the pulse locally destabilizing the membrane as shown for vesicles with increasing surface charge ${ }^{36}$.
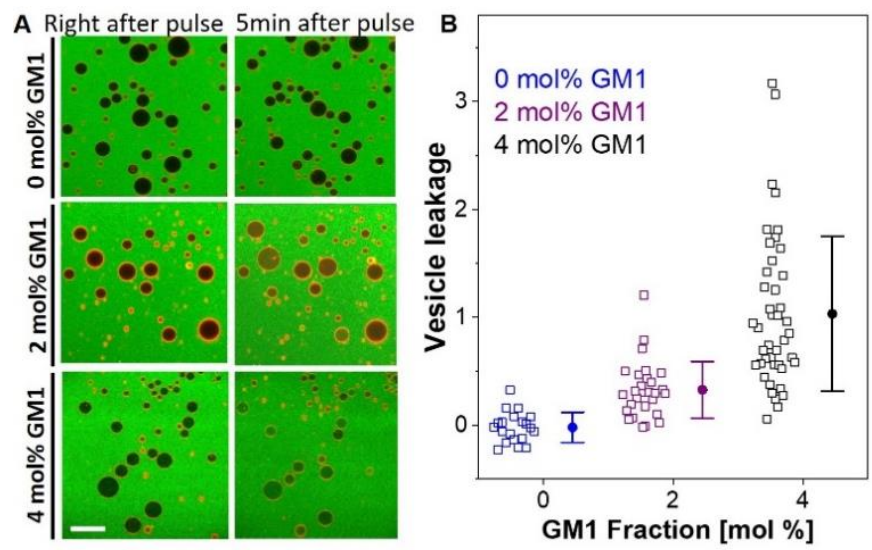

Fig. 3. Long-term permeability of GUVs with increasing fractions of GM1. (A) Confocal images of GUVs illustrating calcein entry into GUVs with increasing fraction of asymmetric GM1 ( 0,2 and $4 \mathrm{~mol} \%$ top to bottom). The medium contained $5 \mu \mathrm{M}$ calcein (green); the GUVs were labeled with $0.1 \mathrm{~mol} \%$ DPPE-Rh (red). The snapshots in the first column were acquired $\sim 4 \mathrm{~s}$ after pulse application $(0.6 \mathrm{kV} / \mathrm{cm}, 3 \mathrm{~ms})$ while the images in the second column show the same vesicles 5 minutes later. The scale bar is $100 \mu \mathrm{m}$. (B) Quantification of GUV leakage through fluorescence intensity analysis; see Eq. 3 in the SI for definition of leakage. Each open symbol corresponds to a measurement on a single GUV. Mean and standard deviation values are shown on the side.

\section{Conclusions}

In summary, we used GM1-doped GUVs to mimic asymmetry of cell membranes. In particular, neuronal cells show elevated concentration of asymmetrically distributed GM1. We observed series of membrane remodeling effects resulting from the electroporation of GM1-containing asymmetric membranes, which all contribute to longer pore lifetimes and partial vesicle destabilization. When the GUV membrane is rendered asymmetric (by dilution), the desorption of GM1 from the outer leaflet of the vesicle membrane triggers the formation of inward tubes stabilized by negative spontaneous curvature. These tubes can physically obstruct the pores and membrane tubulation competes with pore closure, slowing down to the pore closure, reducing the effective membrane edge tension and rendering the membranes permeable at longer timescales. The decrease in edge tension depends on GM1 concentration and degree of leaflet asymmetry. Interestingly, pore lifetimes in the range of tens of seconds have been also observed in GM1-doped membranes but also in the presence of $\mathrm{CTB}^{41}$, which forms homopentamers with $\mathrm{GM} 1^{53}$ in the membrane, presumably leading to even slower reorganization of the pore. Our study also showed that increased fraction of GM1 stabilizes long-living submicroscopic pores and results in leaky vesicles after macropores close. Overall, our findings point to an additional role of GM1 in regulating the integrity of neuronal membranes (which are asymmetrically enriched in GM1) in lowering their stability under electrical perturbation and affecting membrane repair in wound healing. 

available under aCC-BY 4.0 International license.

\section{Acknowledgements}

We thank R. Lipowsky, M. Miettinen and T. Bhatia for fruitful discussions on GM1-doped GUVs. M.A. acknowledges funding from the International Max Planck Research School on Multiscale BioSystems.

\section{Supporting Information}

Vesicle preparation and observation, electroporation, pore stability assessment, edge tension calculation, long-term permeation analysis (including figures with confocal, epifluorescence and phase contrast images as well as corresponding movies, plots for treated data, apparatus set-up for electroporation, analysis of pore stability, deduction of edge tension values, a table for mean edge tension values, quantification of GUV leakage).

\section{Conflict of Interest Disclosure}

The authors declare no conflict of interest.

\section{AUTHOR INFORMATION}

\section{Corresponding Author}

Rumiana Dimova: dimova@mpikg.mpg.de

\section{ORCID IDs}

Mina Aleksanyan: 0000-0002-9817-6244

Rafael Lira: 0000-0001-7362-5827

Jan Steinkühler: 0000-0003-4226-7945

Rumiana Dimova: 0000-0002-3872-8502

\section{Author Contributions}

RD proposed the project. RD, RL and JS designed the experiments. MA, RL and JS performed the experiments and analyzed the data. All authors wrote the manuscript.

\section{REFERENCES}

1. Stewart, M. P.; Langer, R.; Jensen, K. F. Intracellular Delivery by Membrane Disruption: Mechanisms, Strategies, and Concepts. Chemical Reviews 2018, 118, (16), 7409-7531.

2. Yarmush, M. L.; Golberg, A.; Serša, G.; Kotnik, T.; Miklavčič, D. Electroporation-Based Technologies for Medicine: Principles, Applications, and Challenges. Annu. Rev. Biomed. Eng. 2014, 16, (1), 295-320.

$3 . \quad$ Escoffre, J.-M.; Portet, T.; Wasungu, L.; Teissié, J.; Dean, D.; Rols, M.-P. What is (Still not) Known of the Mechanism by Which Electroporation Mediates Gene Transfer and Expression in Cells and Tissues. Molecular Biotechnology 2009, 41, (3), 286295.

4. Dev, S. B.; Rabussay, D. P.; Widera, G.; Hofmann, G. A. Medical applications of electroporation. IEEE Transactions on Plasma Science 2000, 28, (1), 206-223.

5. $\quad$ Frandsen, S. K.; Vissing, M.; Gehl, J. A Comprehensive Review of Calcium Electroporation -A Novel Cancer Treatment Modality. Cancers 2020, 12, (2), 290.

6. Aycock, K.; Davalos, R. Irreversible Electroporation: Background, Theory, and Review of Recent Developments in Clinical Oncology. Bioelectricity 2019, 1, 214-234

7. Ho, M. P., Tissue Engineering with Electroporation. In Handbook of Electroporation, Miklavcic, D., Ed. Springer International Publishing: Cham, 2016; pp 1-21.

8. Davalos, R. V.; Mir, L. M.; Rubinsky, B. Tissue Ablation with Irreversible Electroporation. Annals of Biomedical Engineering 2005, 33, (2), 223.
9. Gothelf, A.; Mir, L. M.; Gehl, J. Electrochemotherapy: results of cancer treatment using enhanced delivery of bleomycin by electroporation. Cancer Treat. Rev. 2003, 29, (5), 371-387.

10. Probst, U.; Fuhrmann, I.; Beyer, L.; Wiggermann, P. Electrochemotherapy as a New Modality in Interventional Oncology: A Review. Technology in Cancer Research \& Treatment 2018, 17, 1-12.

11. Chang, T. T.; Zhou, V. X.; Rubinsky, B. Using nonthermal irreversible electroporation to create an in vivo niche for exogenous cell engraftment. BioTechniques 2017, 62, (5), 229-231. 12. Kotnik, T.; Frey, W.; Sack, M.; Haberl Meglič, S.; Peterka, M.; Miklavčič, D. Electroporation-based applications in biotechnology. Trends in Biotechnology 2015, 33, (8), 480-488.

13. Yadollahpour, A.; Rezaee, Z. Electroporation as a New Cancer Treatment Technique: A Review on the Mechanisms of Action. Biomedical \& Pharmacology Journal 2014, 7, (1), 53-62. 14. André, F.; Mir, L. M. DNA electrotransfer: its principles and an updated review of its therapeutic applications. Gene Therapy 2004, 11, (1), S33-S42.

15. Kim, T. K.; Eberwine, J. H. Mammalian cell transfection: the present and the future. Analytical and Bioanalytical Chemistry 2010, 397, (8), 3173-3178.

16. Mahnič-Kalamiza, S.; Vorobiev, E.; Miklavčič, D. Electroporation in Food Processing and Biorefinery. The Journal of Membrane Biology 2014, 247, (12), 1279-1304.

17. Saulis, G.; Venslauskas, M. S.; Naktinis, J. Kinetics of pore resealing in cell membranes after electroporation. Journal of Electroanalytical Chemistry and Interfacial Electrochemistry 1991, 321, (1), 1-13.

18. Batista Napotnik, T.; Miklavčič, D. In vitro electroporation detection methods - An overview. Bioelectrochemistry 2018, 120, 166-182.

19. Kotnik, T.; Rems, L.; Tarek, M.; Miklavčič, D. Membrane Electroporation and Electropermeabilization: Mechanisms and Models. Annual Review of Biophysics 2019, 48, (1), 63-91.

20. Sengel, J. T.; Wallace, M. I. Imaging the dynamics of individual electropores. Proceedings of the National Academy of Sciences 2016, 113, (19), 5281.

21. Adamo, A.; Arione, A.; Sharei, A.; Jensen, K. F. FlowThrough Comb Electroporation Device for Delivery of Macromolecules. Analytical Chemistry 2013, 85, (3), 1637-1641. 22. Saulis, G.; Saule, R. Size of the pores created by an electric pulse: Microsecond vs millisecond pulses. Biochim. Biophys. Acta-Biomembr. 2012, 1818, (12), 3032-3039.

23. Pakhomov, A. G.; Kolb, J. F.; White, J. A.; Joshi, R. P.; Xiao, S.; Schoenbach, K. H. Long-lasting plasma membrane permeabilization in mammalian cells by nanosecond pulsed electric field (nsPEF). Bioelectromagnetics 2007, 28, (8), 655-663.

24. Dimova, R. Giant Vesicles and Their Use in Assays for Assessing Membrane Phase State, Curvature, Mechanics, and Electrical Properties. Annu. Rev. Biophys. 2019, 48, (1), 93-119.

25. Dimova, R.; Marques, C., The Giant Vesicle Book. Taylor \& Francis Group, LLC: Boca Raton, 2019.

26. Dimova, R.; Riske, K. A.; Aranda, S.; Bezlyepkina, N.; Knorr, R. L.; Lipowsky, R. Giant vesicles in electric fields. Soft Matter 2007, 3, (7), 817-827.

27. Dimova, R.; Bezlyepkina, N.; Jordö, M. D.; Knorr, R. L.; Riske, K. A.; Staykova, M.; Vlahovska, P. M.; Yamamoto, T.; Yang, P.; Lipowsky, R. Vesicles in electric fields: Some novel aspects of membrane behavior. Soft Matter 2009, 5, (17), 32013212 .

28. Perrier, D. L.; Rems, L.; Boukany, P. E. Lipid vesicles in pulsed electric fields: Fundamental principles of the membrane response and its biomedical applications. Adv. Colloid Interface Sci. 2017, 249, 248-271. 
bioRxiv preprint doi: https://doi.org/10.1101/2022.01.21.477228; this version posted January 23, 2022. The copyright holder for this preprint (which was not certified by peer review) is the author/funder, who has granted bioRxiv a license to display the preprint in perpetuity. It is made available under aCC-BY 4.0 International license.

29. Needham, D.; Hochmuth, R. M. Electro-mechanical permeabilization of lipid vesicles. Role of membrane tension and compressibility. Biophysical Journal 1989, 55, (5), 1001-1009.

30. Riske, K. A.; Dimova, R. Electro-Deformation and Poration of Giant Vesicles Viewed with High Temporal Resolution. Biophysical Journal 2005, 88, (2), 1143-1155.

31. Brochard-Wyart, F.; Gennes, P.-G.; Sandre, O. Transient pores in stretched vesicles: role of leak-out. Physica A: Statistical Mechanics and its Applications 2000, 278, 32-51.

32. Karatekin, E.; Sandre, O.; Guitouni, H.; Borghi, N.; Puech, P.-H.; Brochard-Wyart, F. Cascades of Transient Pores in Giant Vesicles: Line Tension and Transport. Biophysical Journal 2003, 84, (3), 1734-1749.

33. $\quad$ Sandre, O.; Moreaux, L.; Brochard-Wyart, F. Dynamics of transient pores in stretched vesicles. Proceedings of the National Academy of Sciences 1999, 96, (19), 10591.

34. Lira, R. B.; Steinkühler, J.; Knorr, R. L.; Dimova, R.; Riske, K. A. Posing for a picture: vesicle immobilization in agarose gel. Scientific Reports 2016, 6, (1), 25254.

35. Mattei, B.; Lira, R. B.; Perez, K. R.; Riske, K. A. Membrane permeabilization induced by Triton X-100: The role of membrane phase state and edge tension. Chemistry and physics of lipids 2017, 202, 28-37.

36. Lira, R. B.; Leomil, F. S. C.; Melo, R. J.; Riske, K. A.; Dimova, R. To Close or to Collapse: The Role of Charges on Membrane Stability upon Pore Formation. Adv. Sci. 2021, 8, (11), 2004068.

37. Lira, Rafael B.; Dimova, R.; Riske, Karin A. Giant Unilamellar Vesicles Formed by Hybrid Films of Agarose and Lipids Display Altered Mechanical Properties. Biophysical Journal 2014, 107, (7), 1609-1619.

38. Sonnino, S.; Mauri, L.; Chigorno, V.; Prinetti, A. Gangliosides as components of lipid membrane domains. Glycobiology 2006, 17, (1), 1R-13R.

39. Sachl, R.; Arnaro, M.; Aydogan, G.; Koukalova, A.; Mikhalyov, I. I.; Boldyrev, I. A.; Humpolickova, J.; Hof, M. On multivalent receptor activity of GM1 in cholesterol containing membranes. Biochimica Et Biophysica Acta-Molecular Cell Research 2015, 1853, (4), 850-857.

40. Chiricozzi, E.; Lunghi, G.; Di Biase, E.; Fazzari, M.; Sonnino, S.; Mauri, L. GM1 Ganglioside Is A Key Factor in Maintaining the Mammalian Neuronal Functions Avoiding Neurodegeneration. International journal of molecular sciences 2020, 21, (3), 868.

41. $\quad$ Dasgupta, R.; Miettinen, M. S.; Fricke, N.; Lipowsky, R.; Dimova, R. The glycolipid GM1 reshapes asymmetric biomembranes and giant vesicles by curvature generation.
Proceedings of the National Academy of Sciences 2018, 115, (22), 5756.

42. Miettinen, M. S.; Lipowsky, R. Bilayer Membranes with Frequent Flip-Flops Have Tensionless Leaflets. Nano Lett 2019, 19, (8), 5011-5016.

43. Portet, T.; Dimova, R. A New Method for Measuring Edge Tensions and Stability of Lipid Bilayers: Effect of Membrane Composition. Biophys. J. 2010, 99, (10), 3264-3273.

44. Ledeen, R. W.; Wu, G. Nuclear sphingolipids: metabolism and signaling. Journal of lipid research 2008, 49, (6), 1176-1186.

45. Lipowsky, R. Spontaneous tubulation of membranes and vesicles reveals membrane tension generated by spontaneous curvature. Faraday Discussions 2013, 161, 305-331.

46. Lipowsky, R., Understanding Membranes and Vesicles: A Personal Recollection of the Last Two Decades. In Physics of Biological Membranes, Bassereau, P.; Sens, P., Eds. Springer International Publishing: Cham, 2018; pp 3-44.

47. Steinkühler, J.; De Tillieux, P.; Knorr, R. L.; Lipowsky, R.; Dimova, R. Charged giant unilamellar vesicles prepared by electroformation exhibit nanotubes and transbilayer lipid asymmetry. Sci. Rep. 2018, 8, (1), 11838.

48. Karimi, M.; Steinkühler, J.; Roy, D.; Dasgupta, R.; Lipowsky, R.; Dimova, R. Asymmetric Ionic Conditions Generate Large Membrane Curvatures. Nano Letters 2018, 18, (12), 78167821 .

49. Liu, Y.; Agudo-Canalejo, J.; Grafmüller, A.; Dimova, R.; Lipowsky, R. Patterns of Flexible Nanotubes Formed by LiquidOrdered and Liquid-Disordered Membranes. ACS Nano 2016, 10, (1), 463-474.

50. Blicher, A.; Wodzinska, K.; Fidorra, M.; Winterhalter, M.; Heimburg, T. The Temperature Dependence of Lipid Membrane Permeability, its Quantized Nature, and the Influence of Anesthetics. Biophys. J. 2009, 96, (11), 4581-4591.

51. Knorr, R. L.; Steinkühler, J.; Dimova, R. Micron-sized domains in quasi single-component giant vesicles. Biochim. Biophys. Acta-Biomembr. 2018, 1860, (10), 1957-1964.

52. Fricke, N.; Dimova, R. GM1 Softens POPC Membranes and Induces the Formation of Micron-Sized Domains. Biophys. J. 2016, 111, (9), 1935-1945.

53. Merritt, E. A.; Sarfaty, S.; van den Akker, F.; L'Hoir, C.; Martial, J. A.; Hol, W. G. Crystal structure of cholera toxin Bpentamer bound to receptor GM1 pentasaccharide. Protein science : a publication of the Protein Society 1994, 3, (2), 166-175. 


\title{
Supplementary Information
}

\section{GM1 asymmetry in the membrane stabilizes pores}

\author{
Mina Aleksanyan, ${ }^{1}$ Rafael B. Lira, ${ }^{1,2}$ Jan Steinkühler ${ }^{1,3}$ and Rumiana Dimova*1
}

\footnotetext{
${ }^{1}$ Department of Theory \& Bio-Systems, Max Planck Institute of Colloids and Interfaces, 14424 Potsdam, Germany.

${ }^{2}$ Current address: Moleculaire Biofysica, Zernike Instituut, Rijksuniversiteit, Groningen, the Netherlands

${ }^{3}$ Current address: Department of Biomedical Engineering, Northwestern University, Evanston, IL 60657, USA

* Address for correspondence: rumiana.dimova@mpikg.mpg.de
}

\section{S1. Preparation of giant unilamellar vesicles (GUVs)}

GUVs were prepared by the electroformation method ${ }^{1,2}$ as described in more detail in Refs. ${ }^{3,4}$ Varying molar fractions of GM1 (Ovine Brain, Avanti Polar Lipids, Alabaster, AL) between 0 and 4 mol\% in 1palmitoyl-2-oleoyl-sn-glycero-3-phosphatidylcholine (POPC) (Avanti Polar Lipids) and $0.1 \mathrm{~mol} \%$ 1,2dipalmitoyl-sn-glycero-3-phosphoethanolamine-N-(lissamine rhodamine B sulfonyl) (DPPE-Rh) (Avanti Polar Lipids) were dissolved in dichloromethane:methanol (2:1) solution to a concentration of $4 \mathrm{mM}$. Both solvents were purchased from Sigma Aldrich, St. Louis, USA. Then, $20 \mu$ of this lipid solution was spread as a lipid film on a pair of indium-tin oxide (ITO)-coated glass plates (PGO GmbH, Iserlohn, Germany), which are electrically conductive and preheated at $50^{\circ} \mathrm{C}$ in the oven. A stream of $\mathrm{N}_{2}$ was used to evaporate most of the solvent, and the plates were subsequently placed under vacuum at $50{ }^{\circ} \mathrm{C}$ for two hours to remove traces of the solvent. This temperature is above the main phase transition temperature of GM1 which is important for ensuring that the membrane is in the fluid and vesicle formation is favored. ${ }^{5}$ For chamber assembly, a Teflon spacer of $2 \mathrm{~mm}$ thickness was placed between the ITO-glass plates and the chamber was filled with $1 \mathrm{mM}$ HEPES buffer ( $\mathrm{pH}$ 7.4, 0.5 Na HEPES; Sigma Aldrich) to hydrate the lipid film. Then, by applying a sinusoidal $A C$ electric field at $10 \mathrm{~Hz}$, electroswelling was initiated at $50{ }^{\circ} \mathrm{C}$. The linear increment of the amplitude from $0.1 \mathrm{~V}$ (peak to peak) to $0.8 \mathrm{~V}$ (peak to peak) during the first $30 \mathrm{~min}$, helping the swelling of the lipids, was followed by a constant application of $0.8 \mathrm{~V}$ for $90 \mathrm{~min}$. Vesicle detachment from the glass surfaces was obtained by decreasing the voltage and frequency gradually to $0.5 \mathrm{~V}$ and $1 \mathrm{~Hz}$ over $60 \mathrm{~min}$. GUVs were slowly cooled to room temperature over $30 \mathrm{~min}$ and then transferred to light-protective glass vials for storage at room temperature. The vesicles were used within 24 hours after preparation.

In order to assess the molar fractions of GM1 on symmetric GUVs, $25 \mu \mathrm{L}$ of $10 \mathrm{mM}$ Alexa Fluor 488 conjugated-cholera toxin B (CTB-Alexa) (Avanti Polar Lipids) were added to $5 \mathrm{~mL}$ of the vesicle solution to reach a final concentration of $50 \mathrm{nM}$ and incubated for $20 \mathrm{~min}$. For intact GUVs, one CTB binds to five GM1 molecules of the outer vesicle leaflet. ${ }^{6}$ The fluorescence intensity of $0-4$ mol\% GM1 containing CTBAlexa labelled GUVs were analyzed through confocal screenshots. The same microscope and objective settings were used during the quantification and generation of statistics for varying concentrations of GM1. Intensity measurements were performed through the "Radial profile extended plugin" from Philippe Carl installed from the ImageJ homepage. The radial intensity profile of GUVs was analyzed at the equatorial plane. A bigger centered circle was drawn on each analyzed vesicle to average the radial intensity in the circular area. An example of a confocal cross section and typical radial intensity profile of a 4 mol\% GM1-doped GUV is plotted in Fig. S1A. The background fluorescence due to free CTB-Alexa dye was subtracted during the data processing and then integrated peak area was taken as the normalized 
intensity for the comparison of GUVs with varying GM1 fractions. Fig. S1B demonstrates the resulting intensity averages of CTB-binding experiments for different GM1 fractions. The data shows a linear trend with the starting GM1 fractions in the membrane.
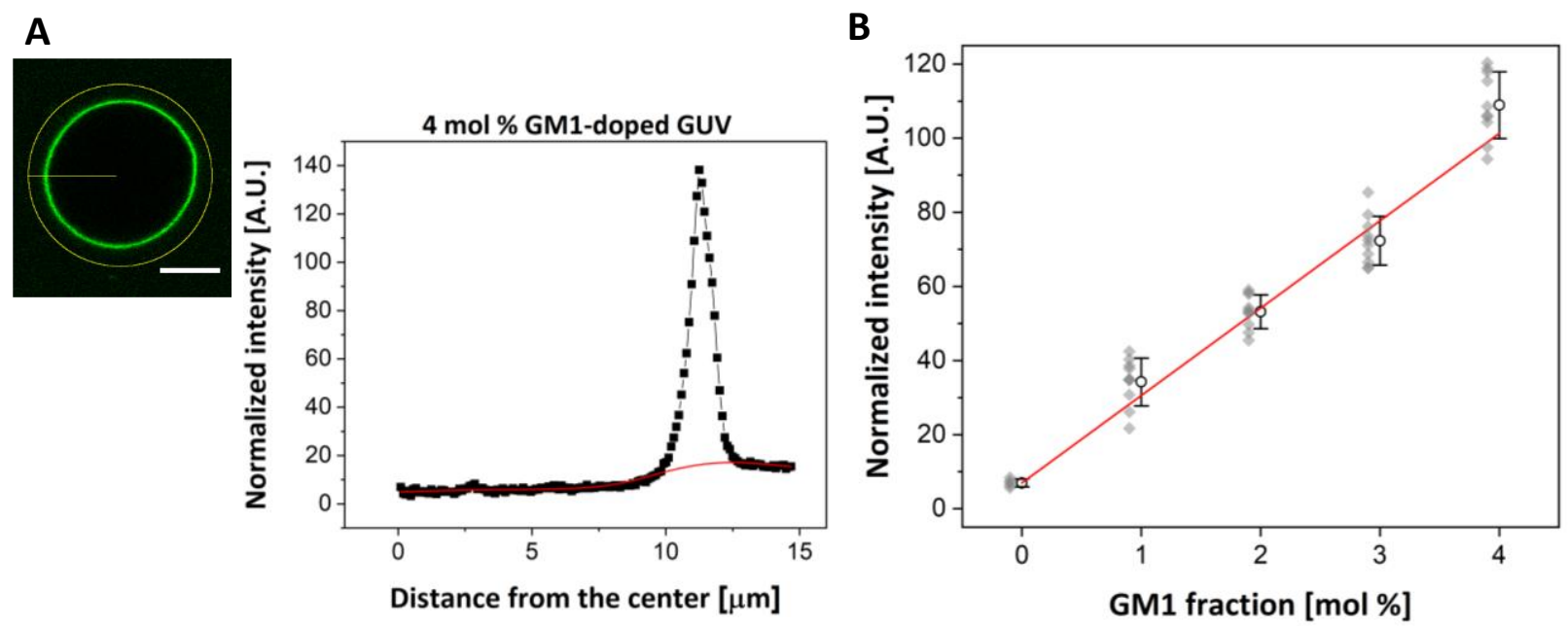

Fig. S1. The presence of GM1 on the outer leaflet of the vesicle membrane was confirmed from fluorescence of CTB-Alexa binding. (A) Representative confocal cross section and radial intensity profile of a symmetric GUV doped with 4 mol\% GM1 analyzed via confocal microscopy and ImageJ radial profile analysis. The scale bar is $10 \mu \mathrm{m}$. Uniform fluorescence signal indicates homogeneous distribution of GM1 along the outer leaflet of the GUV. The graph shows the intensity peak which, after subtraction of the background (red curve) is integrated to quantify the fluorescence due to the binding of CTB-Alexa to the GM1 molecules in the outer leaflet of the GUV. (B) A plot of the resulting intensity averages of CTB-binding experiments for different GM1 fractions. For each GM1 fraction, 10 GUVs from 2 different preparations were analyzed. The data from individual GUVs are shown with light grey diamonds (overlapping single GUV experiments for same GM1 fraction appear as darker gray), while the mean values and standard deviations are displayed with black circles and line bars on the side. The red line shows the linear fit of the normalized mean intensity values for varying GM1 fractions.

\section{S2. Generation of asymmetric GUVs}

We generated GUVs with asymmetric distribution of GM1 in the two membrane leaflets. The asymmetry was established via GM1 desorption achieved with 10-fold dilution of the vesicle media with isotonic GM1free $1 \mathrm{mM}$ HEPES buffer, i.e. the buffer used for vesicle swelling. In order to visually assess the effects of asymmetry, pure POPC GUVs and 4 mol\% GM1-doped GUVs were monitored with confocal laser scanning microscopy in non-diluted and 10-fold diluted conditions. In case of GM1-free vesicles (pure POPC), 100 GUVs in total from 8 independent samples were analyzed for each condition. The vesicles (with diameters above $10 \mu \mathrm{m}$ ) were inspected for defects with 3D confocal scans. In both diluted and non-diluted suspensions, $60 \%$ of the GUVs appeared spherical and defect-free (Fig. S2A). The rest $40 \%$ of the vesicles showed deformed shapes (dumbbell-like structure or tubulation) or had inner GUVs. For GM1-doped membranes, 120 GUVs containing 4 mol\% GM1 from 10 different samples were examined. Before dilution, $80 \%$ of the vesicles were defect-free. As a result of the 10 -fold dilution with isotonic solution, 90 $\%$ of them formed inward tubes (Fig. S2B) stabilized from the negative spontaneous curvature of the asymmetric membrane. Some of the tubes appeared necklace-like with thickness in the suboptical range (Fig. S2C). Their morphology depends on the length and growth kinetics of the tubes, as well as on the elastic properties of GUVs. ${ }^{3,7,8}$ 


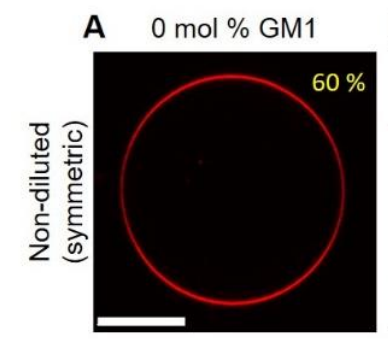

B $4 \mathrm{~mol} \% \mathrm{GM} 1$

C
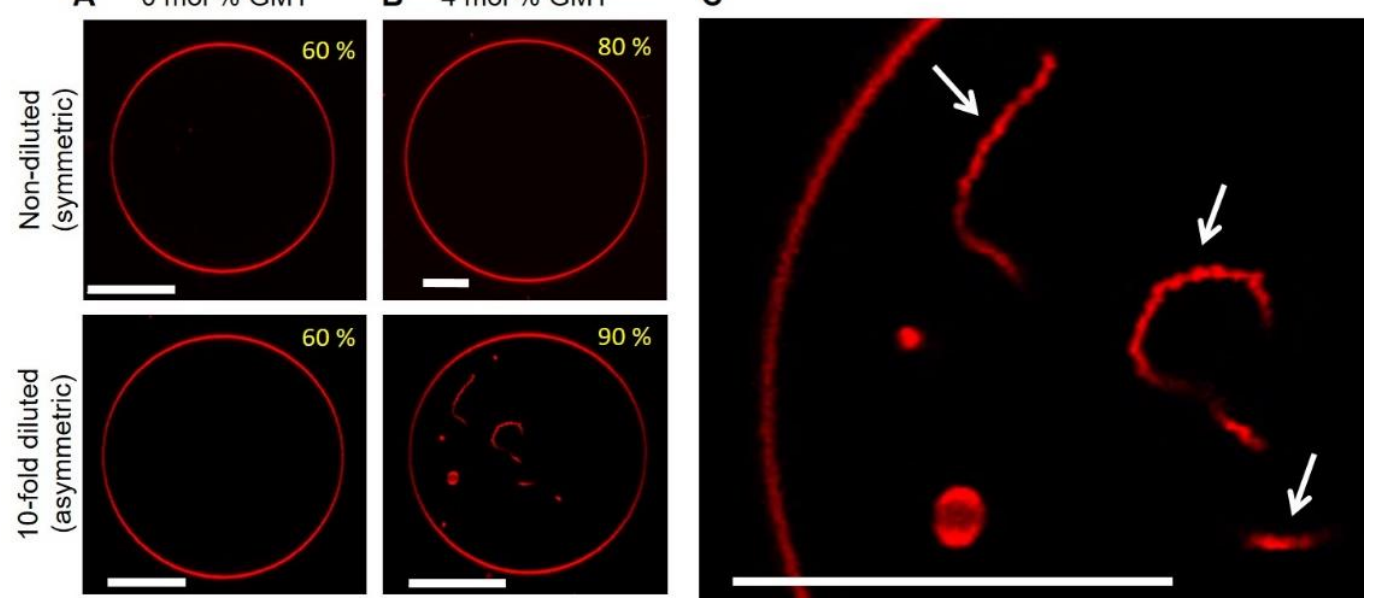

Fig. S2. GM1 desorption upon dilution of GUV external medium generates inward tubes stabilized by negative spontaneous curvature. (A) Confocal cross-sections of pure POPC in non-diluted and 10-fold diluted conditions. (B) Confocal cross-sections of 4 mol\% GM1-doped GUV in non-diluted and 10-fold diluted conditions. In non-diluted conditions, both types of vesicles were smooth without inward or outward structures, i.e. with symmetric membranes. A 10-fold dilution in isotonic and GM1-free $1 \mathrm{mM}$ HEPES solution caused the formation of internal nanotubes. The percentage of defect-free and tubulated vesicles relative to total GUV population is indicated in the upper-left corner of each snapshot. (C) Zoomed-in segment of the GM1-doped GUV in (B, lower panel) showing the necklace-like structure of the inward tubes (arrows). All scale bars are $20 \mu \mathrm{m}$.

\section{S3. GUV Electroporation and imaging}

Electroporation: The GUVs were placed in a home-made electroporation chamber as described by Portet et al. ${ }^{9}$ The chamber was assembled on a $26 \times 56 \mathrm{~mm}$ cover glass equipped with a pair of parallel adhesive copper strips (3M, Cergy-Pontoise, France), $0.5 \mathrm{~cm}$ apart from each other. Then, small parafilm pieces were melted/glued onto the glass slide at a $1 \mathrm{~cm}$ distance from each other to seal the ends. An aliquot of $100 \mu \mathrm{L}$ GUV solution was placed in the space between the copper tapes and a $22 \times 22 \mathrm{~mm}$ coverslip (Thermo Fischer Scientific, Waltham, USA) was placed on top of the solution, forming a closed compartment. (Fig. S3A) Electrodes connected to ßtech pulse generator GHT_Bi500 (ßtech, I'Union, France) were attached to the edges of the copper tapes (Fig. S3B). The field strength and duration of the electric pulses were set to $150-200 \mathrm{~V}$ and $50 \mathrm{~ms}$, respectively. In these pulse parameters, vesicles larger than $10 \mu \mathrm{m}$ (as explored here) porate because the reached transmembrane potential exceeds the critical transmembrane potential for poration. ${ }^{10,11}$ The response of the vesicles to a single (first) DC pulse was recorded for $2-5$ minutes. The procedure was repeated on $10-15$ GUVs for each composition.

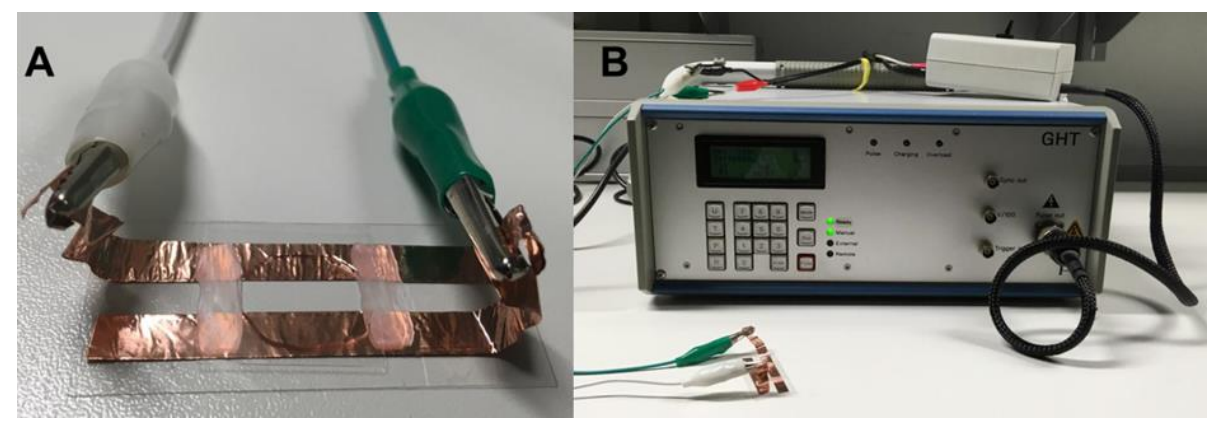

Fig. S3. Illustration of the electroporation set-up. (A) Home-made electroporation chamber. Two adhesive copper strips, $0.5 \mathrm{~cm}$ apart from each other were attached to a cover slide. Small parafilm pieces were placed orthogonally. After the addition of GUV solution, a small coverslip was placed on top and electrodes were connected to the copper strips. (B) Pulse Generator. A single DC pulse was applied (150 - $200 \mathrm{~V}$ and $50 \mathrm{~ms}$ ) in each of the sample after which the sample was discarded. 
GUV imaging with confocal laser scanning microscopy: The morphology of GUVs was monitored through Leica TCS SP5 or SP8 (Wetzlar, Germany) confocal microscopes. Observations were performed either via $40 \times(0.75 \mathrm{NA})$ air or $63 \times(1.2 \mathrm{NA})$ water immersion objectives. In the presence of the green dyes CTBAlexa and calcein, excitation was performed with an argon laser line at $488 \mathrm{~nm}$ and the signal was collected in the range $495-550 \mathrm{~nm}$. The red dye DPPE-Rh was excited with a diode-pumped solid-state laser at $561 \mathrm{~nm}$ and the emission was detected in the range $565-620 \mathrm{~nm}$. The sequential mode of image acquisition was activated to minimize cross-talk. Images were recorded with $512 \times 512$ pixels and scanned with $400 \mathrm{~Hz}$ speed in the bidirectional mode with two line averages. In order to detect electroporation, a resonant scanner was used, which increased the scanning speed to $800 \mathrm{~Hz}$. The images could be obtained on both forward and back scans, allowing to record 36 frames per second (fps). Image analyses were performed through Leica LASX software (Jena, Germany) and ImageJ (NIH, USA). Analyzed data were plotted through Origin Pro software.

Epifluorescence microscopy and high-speed imaging: Edge tension analysis of GUVs during the electroporation experiments was conducted with Axio Observer D1 epifluorescence microscope (Zeiss, Germany) equipped with a sCMOS camera (pco.edge, PCO AG, Kelheim, Germany). Images were recorded in epifluorescence mode with a $40 \times$ / 0.60 Korr Ph2 objective (Zeiss) at a varying acquisition speed between 50-100 fps. The samples were irradiated by using the HBO $100 \mathrm{~W}$ mercury lamp of the microscope in epi-illumination mode. For red irradiation, the light from the mercury lamp passed through $560 / 40,585$, and $630 / 75 \mathrm{~nm}$ filter cube, respectively. The image analyses were performed with the ImageJ software.

Phase contrast microscopy and high-speed imaging: For phase contrast microscopy, GUVs were imaged by a Zeiss Axiovert 200 microscope (Jena, Germany) equipped with an ultra-high-speed digital camera v2512 (Phantom, Vision Research, New Jersey, USA). A halogen lamp HAL 100 was used for the illumination of the samples and all the images were collected with a 40x Ph2 objective at acquisition speed of $5000 \mathrm{fps}$.

\section{S4. Membrane tubulation after pore formation}
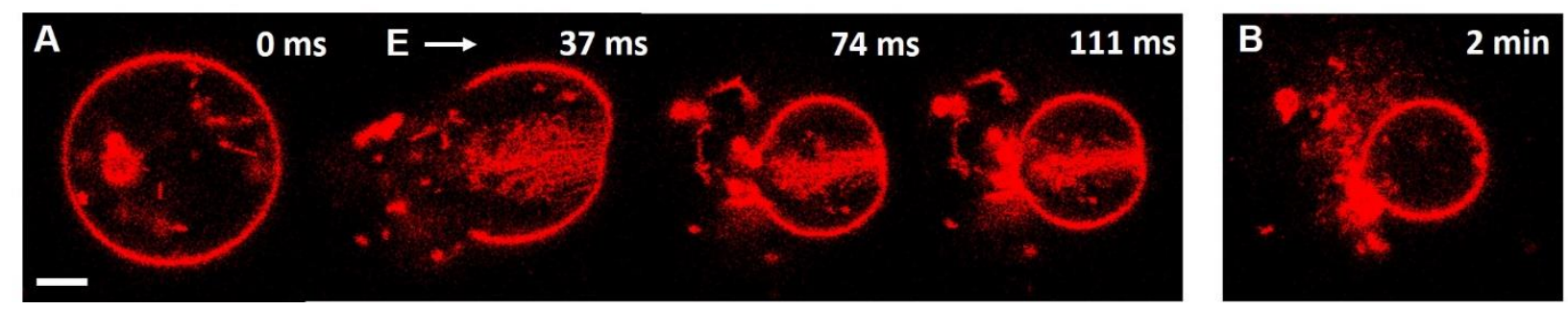

Fig. S4. Sprouting of membrane nanotubes from 4 mol\% GM1-doped asymmetric GUV; also shown in Movie S3. (A) A sequence of images obtained with confocal microscopy during and after application of a DC pulse $(0.3 \mathrm{kV} / \mathrm{cm}, 50 \mathrm{~ms})$. The direction of the electric field is illustrated with the arrow. The time is relative to the beginning of the pulse and shown on the left-upper side of each snapshot. The membrane contains 0.1 mol\% DPPE-Rh and the GUV was 10-fold diluted with 1 mM isotonic HEPES buffer.

(B) Cross section of the vesicle 2 minutes after the pulse application. The scale bar is $10 \mu \mathrm{m}$. 


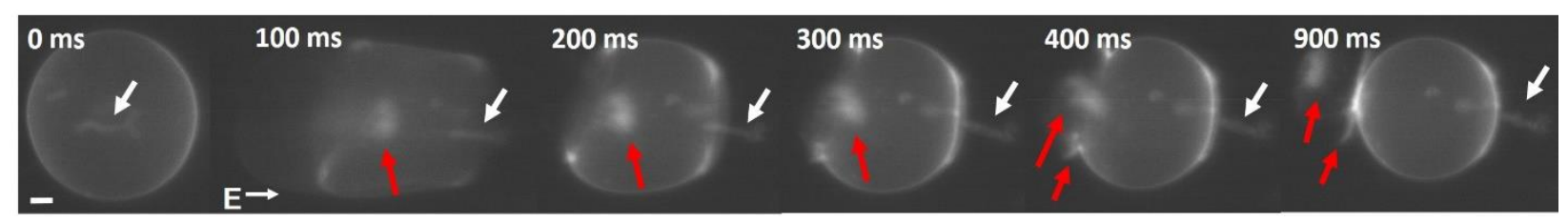

Fig. S5. Sprouting of membrane nanotubes from 4 mol\% GM1-doped asymmetric GUV; also shown in Movie S4. A sequence of images obtained with epifluorescence microscopy during and after the application of a DC pulse $(0.3 \mathrm{kV} / \mathrm{cm}, 50 \mathrm{~ms})$. The direction of the electric field is illustrated with the arrow. The time is relative to the beginning of the pulse and shown on the left-upper side of each snapshot. The white arrow points to an inward tube present already before the application of the pulse. Red arrows point to tubes protruding inside the vesicle and at the pore rims formed after the application of the pulse and expelled outside the vesicle after the application of the pulse. The membrane contains 0.1 mol\% DPPE-Rh and the GUV was 10-fold diluted with 1 $\mathrm{mM}$ isotonic HEPES buffer. The scale bar is $10 \mu \mathrm{m}$.

\section{S5. Pore stability}

We compare the edge energy to the bending energy of the asymmetric membrane as the pore radius increases. When the membrane bending energy is larger than the open pore edge energy, it is unfavorable to close the pore and the vesicle is destabilized whereby the flat membrane would reshape into tubular structure rather than closing the pore (see Fig. S6).

The energy of a porated vesicle of radius $R$ is a sum of the Helfrich and the rim energy:

$E=2 \kappa \int_{A}(M-m)^{2} d A+2 \pi \gamma r$

where $\kappa$ is the membrane bending rigidity, $M=1 / R$ is the vesicle mean curvature, $m$ is the membrane spontaneous curvature (assumed constant), $\kappa_{G}$ is the Gaussian curvature modulus, $r$ is the pore radius and $\gamma$ is the edge tension. The integral in Eq. S1 is over the vesicle (non-porated) area $A=$ $2 \pi R^{2}\left[1+\sqrt{1-(r / R)^{2}}\right]$. Here, we have ignored Gaussian curvature contributions because the topology of a vesicle with expanding pore is preserved.

For a pore to have the tendency to expand, as is the case of asymmetric vesicles, the energy of the system should decrease, i.e.

$d E / d r=-\frac{4 \pi \kappa R(1 / R-m)^{2}}{\sqrt{(R / r)^{2}-1}}+2 \pi \gamma<0$.

Above we assumed that the vesicle radius remains constant, which for small changes in the pore radius is roughly the case. Equation S2 implies that the edge tension term should be dominated by curvature contributions, implying that the tension associated with the spontaneous curvature (spontaneous tension) can act to pull the pore open.

The inequality $\mathrm{S} 2$ can be transformed into

$r>\left(\frac{4 \kappa^{2} m^{4}}{\gamma^{2}}-\frac{1}{R^{2}}\right)^{-1 / 2}$

Considering the large size of the explored GUVs ( $R$ is typically above $20 \mu \mathrm{m}$ ), the second term in the brackets can be ignored, leading to a critical pore size, $r_{c}$, above which the pore would rather expand and the vesicle will collapse

$r>r_{c} \equiv \gamma / 2 \kappa m^{2}$

Introducing the values $\kappa=1.2 \times 10^{-19} \mathrm{~J}$ and $\gamma=17 \mathrm{pN}$ (see Fig. $2 \mathrm{C}$ in the main text) and using $m=-1 / 460$ $\mathrm{nm}^{-1}$ for vesicles prepared with 2 mol\% GM1 and $\gamma=7 \mathrm{pN}$ and $m=-1 / 220 \mathrm{~nm}^{-1}$ for vesicles prepared with 4 mol\% GM1 ${ }^{3}$ we obtain for the critical pore size $r_{c} \sim 15 \mu \mathrm{m}$ and $1.4 \mu \mathrm{m}$ for the respective compositions. 


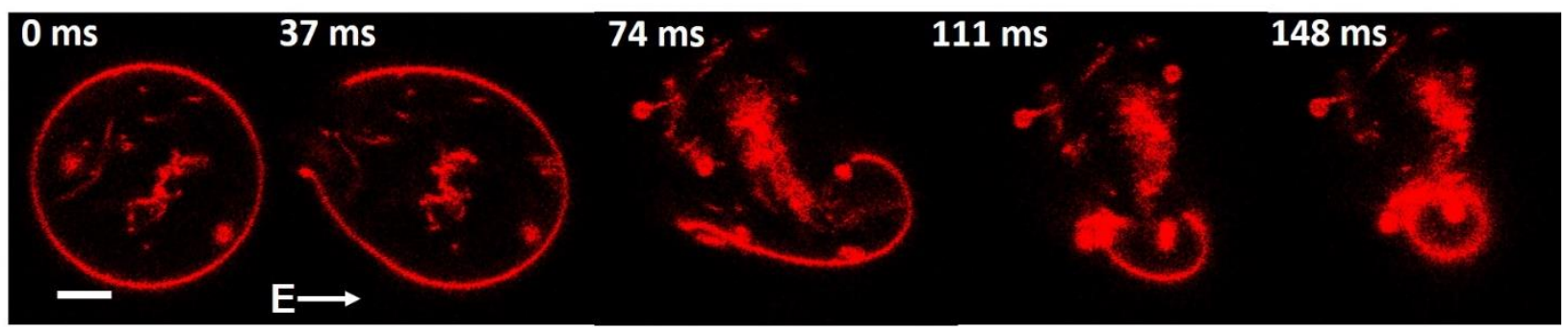

Fig. S6. Complete transformation of 4 mol\% GM1-doped asymmetric GUV to a tubular network. A sequence of images obtained with confocal microscope during and after application of a DC pulse $(0.3 \mathrm{kV} / \mathrm{cm}, 50 \mathrm{~ms})$. The direction of the electric field is illustrated with the arrow. The time is relative to the beginning of the pulse and shown on the left-upper side of each snapshot. The membrane contains 0.1 mol\% DPPE-Rh and the GUV was 10 -fold diluted with $1 \mathrm{mM}$ isotonic HEPES buffer. The scale bar is $10 \mu \mathrm{m}$.

\section{S6. Edge tension analysis of GUVs}

Edge tension measurements are based on the theory described by Brochard-Wyart et al. ${ }^{12,13}$ In order to study in more details the effect of GM1, we measured edge tension for GUVs with increasing GM1 molar fraction. To facilitate the visualization of membrane pores and still have a good temporal resolution, pore closure dynamics were recorded using epifluorescence microscopy. The data were evaluated by manually measuring the pore radius, $r$, from every recorded image. Pore dynamics are characterized by the quantity, $R^{2} \ln (r)$ as a function of time, in which $R$ and $r$ are GUV and pore radius, respectively. Three typical datasets for the different GM1 fractions are shown in Fig. S7A. The linear behavior of the data and the precision of the values of the slopes in the graph for the same GM1 fractions validated the accuracy and the consistency of the experiments with the theoretical model. The straight solid lines represent the linear fits of the quasi-static leakout regime. The edge tension is obtained from the slope of linear fit, $\alpha$, using the equation of $\gamma=-\left(\frac{3}{2}\right) \pi \eta \alpha$, where $\gamma$ and $\eta$ are the edge tension and the medium viscosity $(\eta=$ $0.89 \times 10^{-4} \mathrm{~Pa}$.s for our experimental conditions), respectively. The data differences in the $y$-axis result from the differences in the sizes of the GUVs. GUVs with the same GM1 compositions have different absolute values but similar slopes, which is indicative for similar edge tensions. The obtained values for 0,2 and 4 mol\% of GM1-doped GUVs in non-diluted and 10-fold diluted conditions are displayed in Fig. S7B and the mean values of the edge tensions for each composition are listed in Table S1. Standard error values for the mean edge tension measurements of each fraction are also found as reasonable (less than 15\%), indicating the precision of our experiments. 

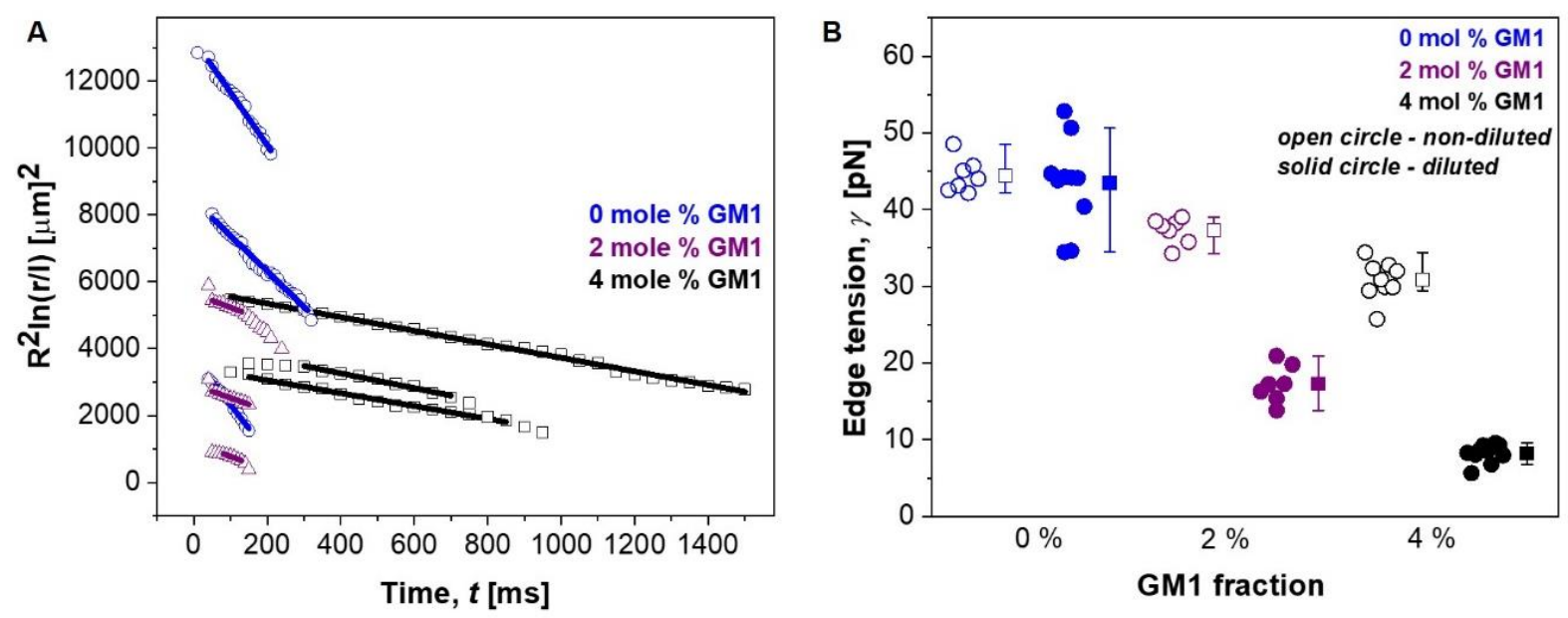

Fig. S7. Pore closure dynamics and edge tension of 0, 2 and 4 mol\% GM1-doped GUVs. (A) Three typical datasets for each composition displayed as the porated region $R^{2} \ln (r)$ versus the time after application of the pulse. Differences in the absolute values of the data come from the differences in GUV radius $R$. In order to avoid plotting a dimensional value in the logarithmic term, the pore radius is scaled by $l=1 \mu \mathrm{m}$. Open symbols reflect the experimental data (each of which required the manual assessment of the pore radius from 20-40 images) while the solid lines are linear fits, the slope of which yield the edge tension (given in panel B). These data show that as the GM1 fraction of GUV increases, the slope decreases. At the same time, the pores display longer lifetime. (B) Edge tension values for vesicles with different molar fractions of GM1 in non-diluted (open circles) and 10-fold diluted (solid circles) systems. Each circle indicates a measurement of an individual GUV. Mean and standard deviation values are shown by the squares and the line bars, respectively. Blue, purple and black corresponds to 0, 2, 4 mol\% of GM1 fractions (same data as in Fig. $2 \mathrm{C}$ in the main text). The results indicate that increasing GM1 fraction and asymmetry decrease membrane edge tension.

Table S1. Mean edge tension values for the GUVs with varying GM1 fraction

\begin{tabular}{ccc}
\multirow{2}{*}{$\begin{array}{c}\text { GM1 } \\
\text { fraction } \\
\text { (mol\%) }\end{array}$} & \multicolumn{2}{c}{ Edge tension (pN) } \\
\cline { 2 - 3 } & $\begin{array}{c}\text { Non-diluted } \\
\text { (symmetric) }\end{array}$ & $\begin{array}{c}\text { Diluted } \\
\text { (asymmetric) }\end{array}$ \\
\hline 0 & $44.4 \pm 2.2$ & $43.3 \pm 5.6$ \\
\hline 2 & $37.2 \pm 1.7$ & $17.2 \pm 2.3$ \\
\hline 4 & $30.8 \pm 2.5$ & $8.2 \pm 1.1$
\end{tabular}

\section{S7. Analysis of GUV prolonged permeability}

The prolonged permeability of GM1-doped asymmetric GUVs after the macropore closure was firstly observed under phase contrast imaging. GM1-doped asymmetric GUVs tended to lose their optical contrast 15 minutes after the macropore closure (Fig. S8) 


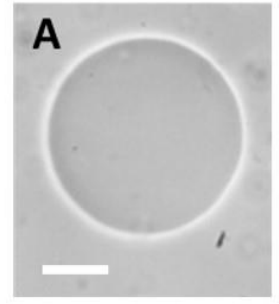

Right after pulse

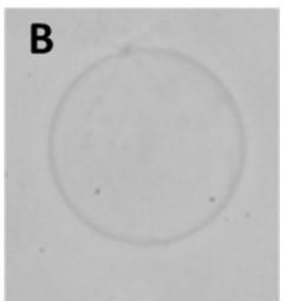

15 min after pulse

Fig. S8. Long-term permeation of 4 mol\% asymmetric GM1-doped GUV right after the macropore closure (A) and 15 minutes after macropore closure (B). The GUV prepared in $200 \mathrm{mM}$ sucrose was 10 -fold diluted in isotonic glucose solution. The contrast loss of the GUV was monitored via phase contrast microscopy after the application of a DC pulse with amplitude of $0.6 \mathrm{kV} / \mathrm{cm}$ and duration of $2 \mathrm{~ms}$. The scale bar is $10 \mu \mathrm{m}$. Due to the different refractive index of the sugar solutions, the vesicles look dark before poration and after the macropore closure. However, during prolonged observations, 15 minutes after the macropore closure, GUV had lost its optical contrast implying that submicroscopic pores remain present.

The long-term permeability of GUVs upon a DC pulse application was quantified by using the small watersoluble marker calcein. An aliquot of $30 \mu \mathrm{L}$ of GUVs prepared in $200 \mathrm{mM}$ sucrose solution ( $200 \mathrm{mosmol} / \mathrm{kg}$ ) was diluted with $65 \mu \mathrm{L}$ isotonic glucose solution and then $5 \mu \mathrm{L}$ of $0.1 \mathrm{mM}$ calcein was added to the diluted system. The sample was incubated for 15 minutes to ensure homogeneity. Then, a single DC pulse with $0.5-0.6 \mathrm{kV} / \mathrm{cm}$ amplitude and $3 \mathrm{~ms}$ duration was applied. Long-term GUV permeation was assessed 5 minutes after the application of the pulse from confocal cross section images of the vesicles in bulk. The calcein influx in GUVs containing $0-4$ mol\% of GM1 was measured from the change in the fluorescence intensity over time in the GUV interior assessing a circular ROI of nominal radius $r_{n}=5 \mu \mathrm{m}$. The fluorescence inside a GUV 5 minutes after the macropore closure was normalized with respect to the fluorescence just after macropore closure and correcting for the fluorescence of the external GUV medium. The GUV leakage, $L$, was defined as:

$$
L=\frac{\left(\frac{F_{\text {in }}}{F_{\text {out }}}\right)_{5}-\left(\frac{F_{\text {in }}}{F_{\text {out }}}\right)_{0}}{\left(\frac{F_{\text {in }}}{F_{\text {out }}}\right)_{0}}
$$

where $F_{\text {in }}$ is the average fluorescence intensity inside the GUV and $F_{\text {out }}$ is the average fluorescence of the medium outside the GUV; the subscripts " 5 " and " 0 " refer to the ratios measured 5 minutes after the macropore closure and right after the macropore closure, respectively. This definition accounts for fluorescence fluctuations due to measurements at different height in the sample (i.e. different vesicle size) or local changes of fluorescence.

The resulting mean intensity values with increasing GM1 fraction were plotted using Origin Pro software. The statistics were generated over $20-40$ GUVs obtained from 3 independent samples. The analyzed GUVs ranged in diameter between 25 and $100 \mu \mathrm{m}$.

\section{Supplementary movies}

Movie S1. A slowed down video of a typical electroporation process of a 4 mol\% GM1-doped asymmetric GUV corresponding to the image sequence shown in Fig. 1E in the main text (where images were rotated 
to keep the same direction of the field in all figures). The sequence was imaged in epifluorescence during and after application of a DC pulse $(0.3 \mathrm{kV} / \mathrm{cm}, 50 \mathrm{~ms})$. The time is relative to the beginning of the pulse and shown on the left-upper side of each snapshot. The membrane contains 0.1 mol\% DPPE-Rh and the GUV was 10 -fold diluted with $1 \mathrm{mM}$ isotonic HEPES buffer. Video was processed with Fiji and slowed down to 8 frames per second.

Movie S2. A slowed down video of a typical electroporation process of a 4 mol\% GM1-doped asymmetric GUV (same vesicle as in Fig. 2 in the main text), presenting a sequence of confocal microscopy images acquired during and after application of a DC pulse $(0.3 \mathrm{kV} / \mathrm{cm}, 50 \mathrm{~ms})$. The time is relative to the beginning of the pulse and shown on the upper left corner of each snapshot. The membrane contains $0.1 \mathrm{~mol} \%$ DPPE-Rh and the GUV was 10-fold diluted with $1 \mathrm{mM}$ isotonic HEPES buffer. The video was processed with Fiji and slowed down to 4 frames per second.

Movie S3. A slowed down video showing membrane nanotubes sprouting and being expelled from a 4 mol\% GM1-doped asymmetric GUV (corresponding to the image sequence shown in Fig. S4 where images were rotated), representing a time sequence of confocal microscopy images during and after application of a DC pulse $(0.3 \mathrm{kV} / \mathrm{cm}, 50 \mathrm{~ms})$. The time is relative to the beginning of the pulse and shown on the left-upper side of each snapshot. The membrane contains $0.1 \mathrm{~mol} \%$ DPPE-Rh and the GUV was 10fold diluted with $1 \mathrm{mM}$ isotonic HEPES buffer. Video was processed with Fiji and slowed down to 3 frames per second.

Movie S4. A slowed down video of sprouting of membrane nanotubes from 4 mol\% GM1-doped asymmetric GUV (corresponding to the image sequence shown in Fig. S5 where images were rotated). A sequence of images obtained in epifluorescence during and after application of a DC pulse $(0.3 \mathrm{kV} / \mathrm{cm}$, $50 \mathrm{~ms}$ ). The time is relative to the beginning of the pulse and shown on the left-upper side of each snapshot. The membrane contains 0.1 mol\% DPPE-Rh and the GUV was 10 -fold diluted with $1 \mathrm{mM}$ isotonic HEPES buffer. Video was processed with Fiji and slowed down to 3 frames per second.

Movie S5. A slowed down video of bursting of 4 mol\% GM1-doped asymmetric GUV to a tubular network shown in Fig. S6. A sequence of images obtained with confocal microscope during and after application of a DC pulse $(0.3 \mathrm{kV} / \mathrm{cm}, 50 \mathrm{~ms})$. The time is relative to the beginning of the pulse and shown on the leftupper side of each snapshot. The membrane contains 0.1 mol\% DPPE-Rh and the GUV was 10-fold diluted with $1 \mathrm{mM}$ isotonic HEPES buffer. Video was processed with Fiji and slowed down to 3 frames per second.

\section{References}

1. Angelova, M. I.; Dimitrov, D. S. Faraday Discussions of the Chemical Society 1986, 81, (0), 303-311. 2. Dimova, R. Annual Review of Biophysics 2019, 48, (1), 93-119.

3. Dasgupta, R.; Miettinen, M. S.; Fricke, N.; Lipowsky, R.; Dimova, R. Proceedings of the National Academy of Sciences 2018, 115, (22), 5756.

4. Bhatia, T.; Agudo-Canalejo, J.; Dimova, R.; Lipowsky, R. ACS Nano 2018, 12, (5), 4478-4485.

5. Walde, P.; Cosentino, K.; Engel, H.; Stano, P. ChemBioChem 2010, 11, (7), 848-865.

6. Merritt, E. A.; Sarfaty, S.; van den Akker, F.; L'Hoir, C.; Martial, J. A.; Hol, W. G. Protein science : a publication of the Protein Society 1994, 3, (2), 166-175.

7. Lipowsky, R. Faraday Discussions 2013, 161, (0), 305-331. 
bioRxiv preprint doi: https://doi.org/10.1101/2022.0121.477228; this version posted January 23, 2022. The copyright holder for this preprint (which was not certified by peer review) is the author/funder, who has granted bioRxiv a license to display the preprint in perpetuity. It is made available under aCC-BY 4.0 International license.

8. Liu, Y.; Agudo-Canalejo, J.; Grafmüller, A.; Dimova, R.; Lipowsky, R. ACS Nano 2016, 10, (1), 463474.

9. $\quad$ Portet, T.; Dimova, R. Biophysical journal 2010, 99, (10), 3264-3273.

10. Needham, D.; Hochmuth, R. M. Biophysical Journal 1989, 55, (5), 1001-1009.

11. Riske, K. A.; Dimova, R. Biophysical Journal 2005, 88, (2), 1143-1155.

12. Brochard-Wyart, F.; Gennes, P.-G.; Sandre, O. Physica A: Statistical Mechanics and its Applications 2000, 278, 32-51.

13. Sandre, O.; Moreaux, L.; Brochard-Wyart, F. Proceedings of the National Academy of Sciences 1999, 96, (19), 10591. 